\title{
The Role of Selenium Nutrition in the Development of Neonatal Rat Lung ${ }^{1}$
}

\author{
H. Y. KIM, M. F. PICCIANO, M. A. WALLIG, AND J. A. MILNER
}

Division of Nutritional Sciences [H.Y.K., M.A.W.], Department of Veterinary Pathobiology [M.A.W.], and School of Human Resources and Family Studies [H.Y.K.], University of Illinois, Urbana, Illinois 61801 and Department of Nutrition, Pennsylvania State University, University Park, Pennsylvania 16802 [M.F.P., J.A.M.]

\begin{abstract}
Our study was designed to assess the role of selenium ( $\mathrm{Se}$ ) in development of neonatal lungs under conditions of normoxia and hyperoxia. Thirty-six female Sprague Dawley rats were bred and fed a Se-deficient $(0.03$ ppm Se) or a Se-adequate $(0.5 \mathrm{ppm} \mathrm{Se})$ diet during pregnancy and lactation. At d 2 postpartum, 24 litters were randomly assigned to either high oxygen $(>95 \%)$ or air and were cross-fostered for $4 \mathrm{~d}$. Lung weight was significantly enhanced in Se-adequate pups and was not related to high oxygen or air exposure of either the pups or dams. Two types of histologic lesions were observed in the lungs of the pups: septal attenuation and interstitial inflammation. When reared in oxygen, all (17 of 17) Se-deficient pups had lesions. In contrast, only $60 \%$ (9 of 15) of Seadequate pups were affected $(p<0.01)$. Lung lesions also were more severe in Se-deficient pups. Se-deficient pups also displayed a significant degree of septal attenuation when reared in air. Se-dependent glutathione peroxidase activity in the pup lung was significantly elevated in response to hyperoxia and was unrelated to Se nutriture. No differences in activities of lung superoxide dismutase, catalase, and glutathione s-transferase were noted between Se-deficient and Se-adequate pups reared in air or high oxygen environments. These data indicate that $\mathrm{Se}$ has an important role in the development of neonatal lungs, a role that is even more pronounced during conditions of hyperoxia. The protective role of $\mathrm{Se}$ in developing lung tissue cannot be completely explained by enhanced glutathione peroxidase activity. (Pediatr Res 29: 440-445, 1991)
\end{abstract}

\section{Abbreviations}

\section{Se, selenium}

LBW, low birth weight

BPD, bronchopulmonary dysplasia

GPx, glutathione peroxidase

SeGPx, Se-dependent GPx

SOD, superoxide dismutase

The newborn of all mammalian species is at risk for the development of Se deficiency because of rapid growth and the variable content of $\mathrm{Se}$ in milk. Se is necessary for growth and fertility in many animals and for prevention of various diseases, such as hepatic necrosis in rats; nutritional myopathies in lambs,

Received March 28, 1990; accepted January 11, 1991

Correspondence: Dr. M. F. Picciano, Pennsylvania State University, S-126 B Henderson Building South, University Park, PA 16802.

Supported in part by NICHHD Grant No. 18689.

' Presented in part at the 14 th International Congress of Nutrition, Seoul, Korea 1989, and the 74th Annual Meeting of the Federation of American Societies for Experimental Biology, Washington, DC, 1990. calves, and goats; and exudative diathesis in chickens (1). Recently, Se deficiency has been recognized as a common occurrence in LBW infants $(2,3)$, with plasma Se values decreasing from 0.75 to $0.35 \mu \mathrm{mol} / \mathrm{L}$ in the first $7 \mathrm{wk}$ of life in LBW infants with respiratory distress syndrome and $\operatorname{BPD}(2,3)$. However, the exact clinical significance of the low Se status in LBW infants is not known at this time.

Each year, substantial numbers of LBW infants develop respiratory distress syndrome, primarily due to immaturity of the lung (4). This reflects surfactant deficiency and structural underdevelopment of the alveoli. Ventilation under high oxygen concentrations is frequently used to treat these premature infants, and this treatment has been associated with the development of a variety of secondary complications including BPD $(5,6)$. Experimentally, high inspired oxygen concentration has been associated with severe functional impairment and morphologic injuries to the lungs of both neonatal and adult rats, mice, and rabbits (7). Many of the histologic changes observed in these animals after exposure to high oxygen resemble those in human infants with BPD.

The cytotoxicity of oxygen is thought to be related to the increased formation of highly reactive oxygen species in the lung during hyperoxia. The lung has several antioxidant defense enzymes, such as SOD, catalase, and GPx, that detoxify the products of oxidative reactions, such as superoxide anion, hydrogen peroxide, and organic hydroperoxides, quite effectively under normal conditions $(8-10)$. By contrast, the increased reactive oxygen species formed during high oxygen inspiration may overwhelm the pulmonary antioxidant defense system and result in pulmonary damage.

Two kinds of GPx are known to exist in the lung. One is SeGPx (EC 1.11.1.9), which reduces both organic and inorganic hydroperoxides (11). The other, known as glutathione s-transferase (EC 2.5.1.18), is not Se-dependent and only detoxifies organic peroxides (12). Se is essential for SeGPx activity (13), and dietary Se modulates the activity of SeGPx in blood and tissues, both in humans and animals $(14,15)$.

Many trace minerals, including Se, are known to accumulate rapidly late in gestation (16). In a similar fashion, the activities of the antioxidant defense enzymes are also known to increase significantly late in gestation (17). Therefore, the low plasma Se concentrations and the low SeGPx activity of LBW infants may be due to a short gestational period. Presently, both parenteral and enteral formulas administered to LBW infants contain no supplemental Se, and whether enhancement of Se status of LBW infants affords them any clinical advantage has not been studied. Because Se concentrations are already low in LBW infants, it is possible that the low SeGPx activities that result may predispose these infants to lung damage and subsequent development of BPD during oxygen treatment. Although the only known role of $\mathrm{Se}$ is that of an essential component of SeGPx, Se is thought to have additional but as yet unidentified functions in normal growth and maintenance (18). It is also unknown whether Se has 
a role, not associated with SeGPx, in development of the neonatal rat lung.

The lungs of many neonatal animals, including rats, have characteristics similar to those of human infants (19). For example, there is incomplete development of rat alveoli at birth, followed by rapid alveolarization of terminal airways after birth. In addition, antioxidant enzyme activities are lower in the neonatal rat than in the adult rat, a characteristic similar to that in the human neonate (20). These developmental similarities make the neonatal rat a suitable model for investigating the role of $\mathrm{Se}$ in development of the neonatal lung under conditions of normoxia and hyperoxia. Our study used this model and was designed to assess the role of Se in neonatal lung development under conditions of normoxia and hyperoxia.

\section{MATERIALS AND METHODS}

Animal care. Nulliparous female, Sprague Dawley rats (Harlan Industries, Indianapolis, IN), weighing 160 to $180 \mathrm{~g}$, were housed in individual, suspended, stainless steel, wire-mesh cages in a room with controlled temperature $\left(20-22^{\circ} \mathrm{C}\right)$ and lighting $(12 \mathrm{~h}$ light-dark cycle). The animals were fed a commercial ration (Purina Rodent Chow, Ralston Purina Co., St. Louis, MO) for a 2- to 3-wk adaptation period. At 200-240 g, the rats were mated, and $d 1$ of pregnancy was determined by the presence of vaginal plugs and sperm. On d 1 of pregnancy, rats were randomly assigned to one of two experimental diets: Se-deficient and Seadequate. Demineralized water (Nanopure; Barnstead, Boston MA) and experimental diets were fed ad libitum. The Animal Care and Use Committee of the University of Illinois reviewed and approved the use of experimental animals in these studies.

Diets. Diets (Table 1) were formulated to contain all nutrients in quantities adequate for reproduction (21), except for Se in the Se-deficient diet. Direct analysis confirmed dietary Se contents of $0.03 \mathrm{ppm}$ (Se-deficient) and $0.5 \mathrm{ppm}$ (Se-adequate).

Experimental design. Thirty-six female rats were bred and fed the Se-deficient $(0.03 \mathrm{ppm})$ or the Se-adequate $(0.5 \mathrm{ppm})$ diet during pregnancy and lactation. Food dishes were positioned so that only dams had access to food, and maternal milk was the only nourishment for pups. The day after parturition (d 2), litters were culled to seven pups per dam. This procedure was followed so that the nutritional burden of dams and milk provision to pups were similar across replicates and treatments. Day 2 was

Table 1. Diet composition

\begin{tabular}{lc}
\hline \multicolumn{1}{c}{ Ingredient } & Amount $(\mathrm{g} / \mathrm{kg})$ \\
\hline Casein* & 200 \\
Sucrose & 300 \\
Cornstarch & 331 \\
Corn oil & 100 \\
Cellulose $\dagger$ & 20 \\
Vitamin mixture $\ddagger$ & 10 \\
Se-free AIN-76 mineral mixture $\$$ & 35 \\
DL-Methionine & 2 \\
Choline bitartrate & 2 \\
\hline
\end{tabular}

*Vitamin-free test casein (Teklad, Madison, WI).

$\dagger$ Alphacel, ICN Nutritional Biochemical Co., Cleveland, $\mathrm{OH}$

‡AIN-76 vitamin mixture. Composition of vitamin mix ( $\mathrm{mg} / \mathrm{kg}$ diet): thiamin $\mathrm{HCl}, 6.0$; riboflavin, 6.0 ; pyridoxine $\mathrm{HCl}$ (vitamin $\mathrm{B}_{6}$ ), 7.0 ; nicotinic acid, 30.0; calcium pantothenate, 16.0; folic acid, 2.0; biotin, 0.2 ; vitamin $B_{12}, 0.01$; vitamin $K, 0.05$. Supplies in $\mathrm{IU} / \mathrm{kg}$ diet: vitamin A, 4000 ; vitamin D, 1000 ; vitamin E, 50.0 ; sucrose, $9.9 \mathrm{~g}$.

$\S$ AIN-76 mineral mixture prepared without Se. Composition of mineral mix $(\mathrm{g} / \mathrm{kg}$ mineral mix): calcium phosphate, dibasic, $500.0 ; \mathrm{NaCl}$, 74.0 ; potassium citrate, monohydrate, 220.0 ; potassium sulfate, 52.0 ; magnesium oxide, 24.0; manganous carbonate, 3.5; ferric citrate, 6.0 ; zinc carbonate, 1.6 ; cupric carbonate, 0.3 ; potassium iodate, 0.01 ; chromium potassium sulfate, 0.55 ; sucrose, 118.05 . Sodium selenite was added to provide $0.5 \mathrm{ppm} \mathrm{Se}$ in formulation of the Se-adequate diet. chosen because dams often reject their litters if they are disturbed on $\mathrm{d} 1$. Two litters from dams delivering pups on the same day were mixed; half of the pooled litter was given to one of these dams and the remaining half to the other dam. The two matched dams were then rotated between their air- and oxygen-housed litters for the next $4 \mathrm{~d}$. In the latter environment, exposure to oxygen was conducted with careful monitoring of oxygen concentration $(>95 \%)$, temperature $\left(22-25^{\circ} \mathrm{C}\right)$, and humidity $(55-$ $75 \%$ ). This exposure to oxygen was continuous during $4 \mathrm{~d}$, except for a brief daily period when chambers were opened for rotation of dams between oxygen- and air-housed litters. This rotation was done to prevent oxygen-induced illness in the dams.

The remaining 12 litters (six litters in each dietary group) stayed with their parent dams in an air environment throughout the experimental period and were used as negative controls for the cross-fostering procedure. Animals were weighed and killed at $\mathrm{d} 6$ postpartum, heparinized blood was collected, and lungs and liver were excised.

Analytical procedures. Two to three pups per litter were used for lung histopathology. Lungs were fixed via intratracheal instillation of $10 \%$ buffered formalin at an inflation pressure of approximately $20 \mathrm{~cm}$ of $\mathrm{H}_{2} \mathrm{O}$. Three standard cross-sections from each lung (left and right) were examined. These sections were taken from the same area of apical, middle, and diaphragmatic portions of each lung. Sections were embedded in paraffin, sectioned at $5 \mu \mathrm{m}$, and stained with hematoxylin and eosin. Sections were examined at low $(10 \times)$ and high $(40 \times)$ magnification by light microscopy to determine if two lesion patterns were present: septal attenuation and interstitial inflammation. The histolic scoring was done in a double-blinded manner. Then, each lesion received a score ranging from 0 (no lesion) to 3 (severe lesion). For septal attenuation, a score of 1 (mild lesion) was recorded when there were only mild, occasional foci of septal bud stunting: a score of 2 (moderate lesion) when there were multiple, diffusely scattered but still mild foci of septal bud stunting; and a score of 3 (severe lesion) when there were many coalescing foci of moderate septal stunting. For inflammatory lesions, a score of 1 (mild lesion) was recorded if there were occasional, small foci of inflammation and interstitial thickening occupying less than $10 \%$ of the lung section; a score of 2 (moderate lesion) if there were multiple foci of inflammation occurring in more than $10 \%$ but less than $50 \%$ of the section; and a score of 3 (severe lesion) if there were multiple coalescent foci of moderately severe inflammation occupying more than $50 \%$ of the alveolar tissue in a section.

Whole blood was centrifuged $(800 \times g)$ and plasma was collected. Lungs were carefully perfused through the pulmonary artery with ice cold isotonic buffer $(0.1 \mathrm{M}$ potassium phosphate, $0.15 \mathrm{M} \mathrm{KCl}, \mathrm{pH} 7.4$ ). Lungs and liver were surgically removed, rinsed, and blotted dry. Lungs and liver from four pups in each litter were kept on ice and weighed on an analytical balance accurate to $0.1 \mathrm{mg}$. Mean lung or liver weight per litter was the unit used for statistical analysis. The tissue samples were pooled, homogenized in ice cold $5 \mathrm{mM}$ phosphate buffer $(\mathrm{pH} 7.8)$, and used for subsequent biochemical assays.

Se concentrations in diet, lung, plasma, and liver were determined according to the method of McCarthy et al. (22), using a gas chromatograph equipped with an electron capture detector (5710A; Hewlett-Packard Co., Avondale, PA) and a 0.53-mm inner diameter fused silica capillary column (Supelco, Bellefonte, PA). The activities of SeGPx and total peroxidase were determined in blood and tissue homogenates by the coupled assay of Paglia and Valentine (23) as modified by Levander et al. (24). This assay used hydroperoxide and $t$-butyl hydroperoxide as substrates. In the lung, the activity of SOD was determined by the inhibition of xanthine- and xanthine oxidase-catalyzed reduction of ferricytochrome $C(25)$. Catalase activity was measured by the Holmes and Masters method (26). Protein content of tissues and blood was determined by a modified Lowry method (27). 
Statistics. Biochemical data and lung histopathologic scoring were evaluated using analysis of variance $(2 \times 2$ factorial $)$ statistics followed by the least significant difference test. Comparisons among groups of data on lung injury were made by the Fisher exact probability test. The value of $p<0.05$ was chosen as the level of statistical significance $(28,29)$.

\section{RESULTS}

Maternal feed intake, body and organ weights, and tissue Se concentrations. Throughout the study, no significant differences in mean feed intake or body and organ weights of dams were observed among groups. The mean feed intake of all dams was $14.2 \pm 0.4 \mathrm{~g} / \mathrm{d}$. The mean body weight $(\mathrm{g})$ of dams was $279.5 \pm$ 3.1 at the end of pregnancy and $198.3 \pm 2.8$ at the end of $6 \mathrm{~d}$ of lactation. The mean lung and liver weights $(\mathrm{g})$ of dams were $1.11 \pm 0.10$ and $6.86 \pm 0.40$, respectively.

Feeding the marginally Se-deficient diet during pregnancy and lactation resulted in significant $(p<0.001)$ decreases in $\mathrm{Se}$ concentrations in dam plasma, liver, and lung. Mean plasma Se concentrations $(\mu \mathrm{mol} / \mathrm{L})$ of Se-deficient and Se-adequate dams were $5.02 \pm 0.22$ and $6.03 \pm 0.15$, respectively; mean liver Se concentrations $(\mu \mathrm{mol} / \mathrm{kg})$ were $9.15 \pm 0.11$ and $14.25 \pm 0.58$, respectively; and mean lung Se concentrations $(\mu \mathrm{mol} / \mathrm{kg})$ were $4.21 \pm 0.36$ and $7.05 \pm 0.37$, respectively.

Body and organ weight of pups. Mean total body, lung, and liver weights of $d 6$ rat pups are shown in Table 2. The mean lung weight of Se-adequate pups was significantly greater than that of Se-deficient pups $(p<0.05)$, regardless of whether pups were reared in oxygen or air. However, the low Se treatment had no effect on either whole body or liver weights of $\mathrm{d} 6$ pups.

Histopathology. All Se-deficient pups (17 of 17) reared in oxygen had lung lesions, whereas only $60 \%$ ( 9 of 15) of Seadequate pups exposed to oxygen had lesions $(p<0.01)$. Typical microscopic characteristics of lungs from the experimental rat pups are shown in Figures 1 and 2. Most lungs from air control pups (Fig. 1). had many small alveoli and numerous septal "buds." After oxygen exposure, two patterns of lung lesions were observed: 1 ) septal attenuation and 2) interstitial inflammation. The alterations associated with septal attenuation included stunting of septal buds, a decrease in the number of alveolar septa, thinning of remaining septal buds, and enlarged alveolar spaces (Fig. $2 A$ ). In lungs with interstitial inflammation (Fig. $2 B$ ), there were multiple foci of thickening and hypercellularity of alveolar septa, mainly due to infiltration by macrophages and some neutrophils. These changes were seldom diffuse but usually patchy and irregular in distribution. Some oxygen-exposed rats had both lesions, others had only one lesion, and others had no lesions at all.

The histopathologic scoring of the lung lesions indicated that interstitial inflammation was more severe in Se-deficient pups under conditions of hyperoxia than in Se-adequate pups under the same conditions $(p<0.05)$ (Table 3$)$. Se-deficient pups reared in oxygen also had significantly more severe septal attenuation in comparison to the pups reared in air, whereas the Seadequate pups reared in oxygen had septal attenuation intermediate in severity. Pups reared in air had few lesions, and the lesions present were significantly milder than those in rats reared in oxygen, regardless of Se status. No dams had lung lesions, regardless of Se status and/or oxygen exposure.

Se concentrations, protein contents, and enzyme activities in pup organs and plasma. Tissue and plasma Se concentrations and SeGPx activities in pups are shown in Table 4. Lung Se concentration and SeGPx activity of d 6, air-exposed pups were similar, regardless of the Se nutriture of the parent dams. On the other hand, when pups were reared in oxygen, the activity of lung SeGPx was significantly elevated in both Se-deficient and Se-adequate groups, but lung Se concentration was not changed. Pup liver and plasma Se concentrations and SeGPx activities were significantly depressed in Se-deficient groups, regardless of air or oxygen exposure.

No differences in activities of lung superoxide dismutase, catalase, and non-Se-dependent GPx (calculated by subtraction of SeGPx from total GPx activity) were observed between Sedeficient and Se-adequate pups in either air or oxygen environment.

Assessment of possible effects of cross-fostering procedure. To assess the cross-fostering procedure as a possible confounding variable, comparisons among cross-fostered and negative control pups reared in air by Se-deficient and Se-adequate dams were made (Table 5). Within the same dietary treatment, crossfostered pups did not show differences in lung weight, Se content, and SeGPx activity compared to negative control pups. Therefore, cross-fostering of pups for $4 \mathrm{~d}$ had no measurable influence on lung growth of pups. However, Se-deficient pups reared in air had reduced lung weight and a significant degree of septal attenuation, whether they remained with parent dams or were crossfostered.

\section{DISCUSSION}

Se is necessary for growth and for prevention of various diseases. In our study, we found that Se also has an important role in the normal development of neonatal lungs and can protect neonates from pulmonary damage under conditions of hyperoxia. The primary effect of Se on pulmonary development is evident from the significantly enhanced lung weights of Seadequate pups whether exposed to oxygen or not and the observation that Se-adequate pups exhibited an advanced stage of alveolar development (less or no septal attenuation) compared with Se-deficient pups reared in air. The protective role of Se is supported by significantly decreased lung damage $(p<0.01)$ of Se-adequate pups exposed to oxygen and less severe lesions compared with Se-deficient pups. In fact, all Se-deficient pups exposed to hyperoxic conditions had lung lesions, whereas only $60 \%$ of Se-adequate pups were affected.

Results from this study also indicate that developing lung may preferentially utilize Se over other tissues and that under conditions of hyperoxia, such utilization is accelerated. Although maternal lung Se content in the Se-deficient group was lower than in the Se-adequate group, pulmonary Se content in pups did not show the same pattern. There was a trend, albeit not statistically significant, for increased lung Se in Se-deficient pups. Furthermore, despite significantly lower liver and plasma Se concentrations and SeGPx activities in Se-deficient pups, pulmonary SeGPx was not significantly lowered under normoxic

Table 2. Mean body, lung, and liver weights of Se-deficient and Se-adequate pups reared in air or oxygen environment*

\begin{tabular}{|c|c|c|c|c|}
\hline & \multicolumn{2}{|c|}{ Se-deficient } & \multicolumn{2}{|c|}{ Se-adequate } \\
\hline & Air & Oxygen & Air & Oxygen \\
\hline Whole body (g) & $8.78(0.54)$ & $8.61(0.35)$ & $9.06(0.25)$ & $9.17(0.17)$ \\
\hline Lung $(\mathrm{g}) \dagger$ & $0.144(0.011)$ & $0.141(0.009)$ & $0.183(0.010)$ & $0.166(0.009)$ \\
\hline Liver $(\mathrm{g})$ & $0.290(0.010)$ & $0.286(0.007)$ & $0.264(0.009)$ & $0.29(0.010)$ \\
\hline
\end{tabular}

$*$ Values are means $( \pm$ SEM) for six litters.

$\dagger$ Significant effect of dietary $\operatorname{Se}(p<0.01)$ by $2 \times 2$ factorial analysis of variance. The coefficients of variance of the lung weight measurement for Se-deficient and Se-adequate groups exposed to air or oxygen are 18.75,15.60,13.11, and $13.25 \%$, respectively. 


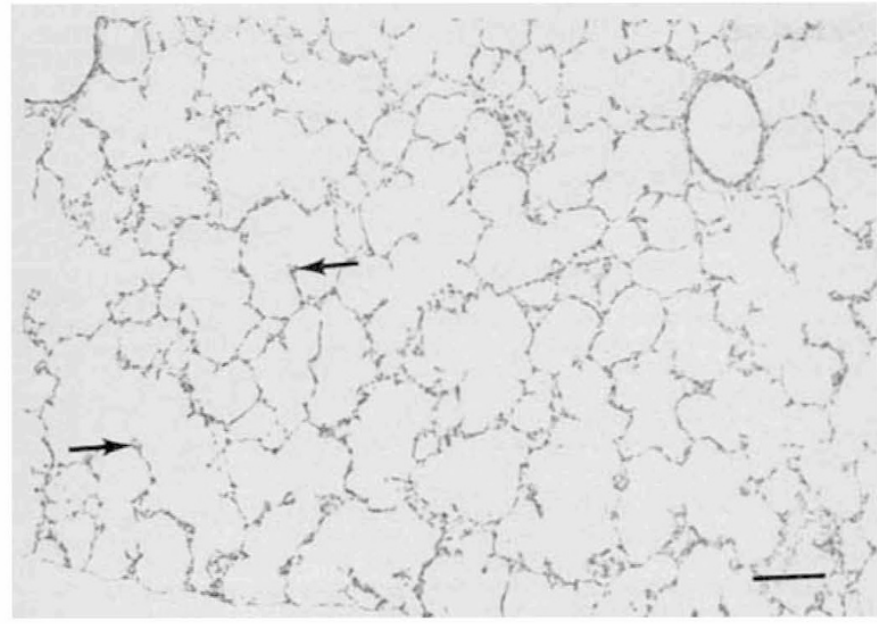

Fig. 1. Lung from a Se-deficient neonatal rat pup exposed to room air for $4 \mathrm{~d}$. The lung has many small alveoli and numerous septal buds (arrows). Hematoxylin and eosin, bar $=60 \mu \mathrm{m}$.

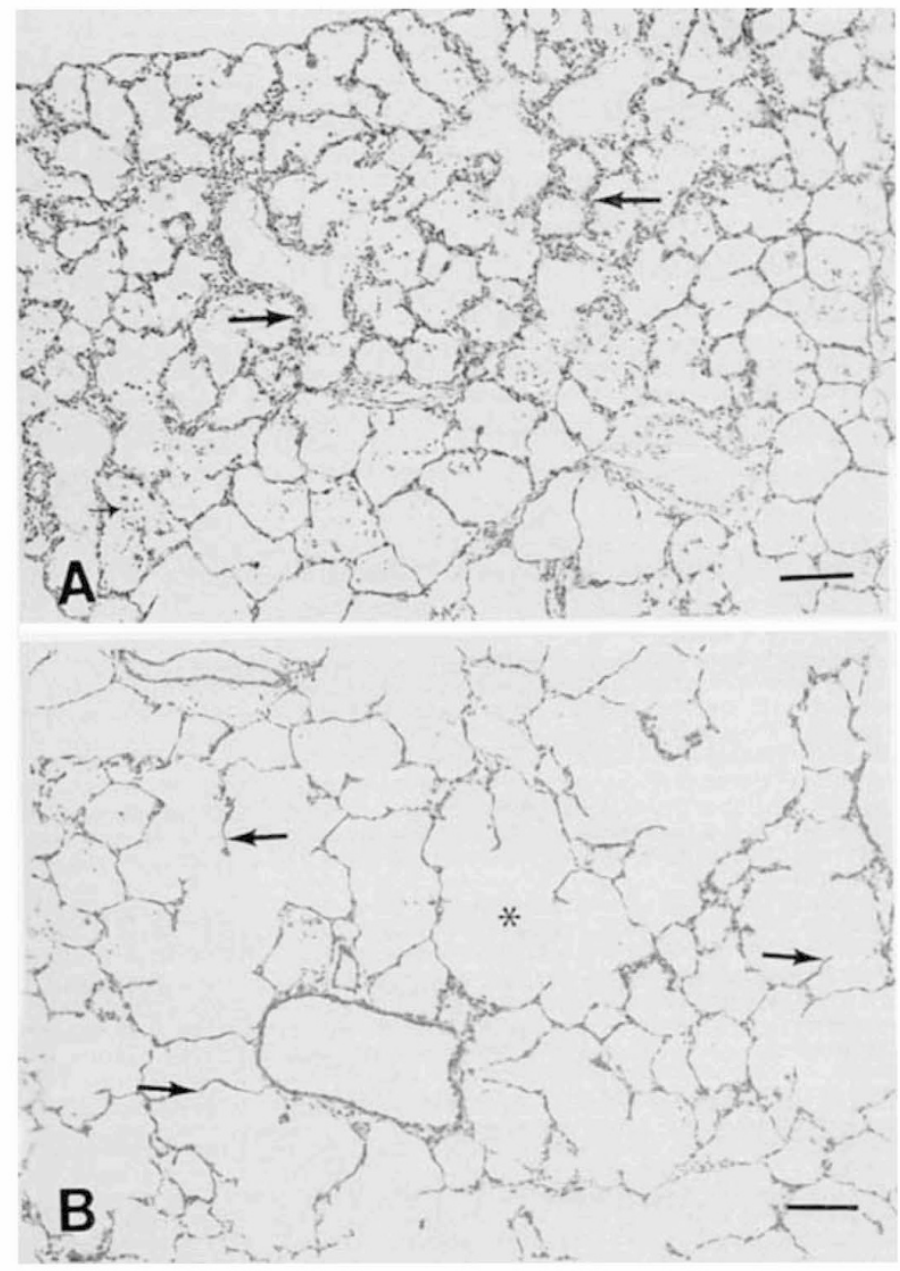

Fig. 2. Lungs from Se-deficient rat pups exposed to $>95 \% \mathrm{O}_{2}$ for 4 d. Hematoxylin and eosin, bar $=60 \mu \mathrm{m}$. A, lung with interstitial inflammation. There is prominent thickening and hypercellularity of alveolar septa (large arrows), mainly due to infiltration by inflammatory cells. There are also increased alveolar macrophages (small arrow) and other inflammatory cells within alveolar spaces. $B$, lung with septal attenuation. In this field, alveolar sacs are much enlarged (*). There are fewer and thinner septal buds (arrows).
Table 3. Histopathologic scoring of lungs from Se-deficient and Se-adequate pups reared in air or oxygen environment

\begin{tabular}{lrrrrr}
\hline & \multicolumn{2}{c}{ Se-deficient } & & \multicolumn{2}{c}{ Se-adequate } \\
\cline { 5 - 6 } & Air & Oxygen & & Air & Oxygen \\
\hline $\begin{array}{l}\text { Interstitial inflam- } \\
\quad \text { mation* }\end{array}$ & & & & & \\
$\quad$ No lesions & 15 & 3 & & 15 & 8 \\
$\quad$ Mild lesions & 2 & 7 & & 2 & 5 \\
$\quad$ Moderate lesions & 0 & 4 & & 0 & 0 \\
$\quad$ Severe lesions & 0 & 3 & & 0 & 2
\end{tabular}

Mean severity $0.12(0.08) \quad 1.41(0.24) \quad 0.12(0.08) \quad 0.73(0.27)$ score† $\S$

Septal attenuation*

No lesions

Mild lesions

Moderate lesions

Severe lesions

Mean severity score $\$$

* Values are numbers of pups with the indicated degree of lesion severity.

$\dagger$ Each lesion received a score ranging from 0 (no lesion) to 3 (severe lesion) for the purpose of statistical analysis $(2 \times 2$ factorial analysis of variance). Values are means for $15-20$ pups ( \pm SEM).

$\ddagger$ Significant effect of dietary Se and significant interaction between dietary Se and oxygen environment.

$\S$ Significant effect of oxygen environment.

Table 4. Mean lung, liver, and plasma Se concentrations and SeGPx activities of Se-deficient and Se-adequate pups reared in air or oxygen environment*

\begin{tabular}{cccccc}
\hline & \multicolumn{2}{c}{ Se-deficient } & & \multicolumn{2}{c}{ Se-adequate } \\
\cline { 2 - 3 } \cline { 5 - 7 } & Air & Oxygen & & Air & Oxygen \\
\hline
\end{tabular}

Selenium con-

centration

$(\mu \mathrm{mol} / \mathrm{kg})$

Lung

Livertit

$\begin{array}{llll}1.62(0.12) & 1.88(0.06) & 1.86(0.11) & 1.75(0.09)\end{array}$

Plasmatts

$2.28(0.19) \quad 2.32(0.15) \quad 3.74(0.21) \quad 2.97(0.15)$

SeGPx activity

(U)\|

$\begin{array}{lllll}\text { Lung§ } & 40.3(3.5) & 57.4(3.3) & 48.2(3.4) & 55.2(4.4) \\ \text { Liver } \dagger & 25.5(2.6) & 21.0(3.5) & 46.4(4.9) & 41.2(3.8) \\ \text { Plasma } \dagger & 17.4(2.3) & 14.1(2.1) & 25.9(2.1) & 21.9(2.3)\end{array}$

$*$ Values are means $( \pm$ SEM) for six litters.

$\dagger$ Significant effect of dietary Se.

$\ddagger$ Significant interaction of dietary Se and oxygen environment. $\S$ Significant effect of oxygen environment.

\|I In $\mu \mathrm{mol} \mathrm{NADPH}$ disappearance $/ \mathrm{min} / \mathrm{g}$ protein.

conditions and, in response to hyperoxia, rose to a level comparable to that of Se-adequate pups.

During high oxygen exposure, extensive functional and morphologic lung damage followed by death can occur in experimental animals. The toxic effects of oxygen on the lung vary depending on the age of the animals (30), concentration of oxygen, and duration of oxygen exposure $(31,32)$. At $>95 \%$ oxygen concentrations, adult animals die from severe lung damage in 3 to $5 \mathrm{~d}\left(\mathrm{LD}_{50}\right)$. Neonatal rats, mice, and rabbits, however, show a higher resistance to oxygen, and most of them survive 7 $\mathrm{d}$ of oxygen exposure (7). In this study, we used 2-d-old neonatal rats as experimental animals. We found no deaths after $4 \mathrm{~d}$ of $>95 \%$ oxygen exposure and we found histologic lesions, such as interstitial inflammation and septal attenuation (retarded alveolar development), that were similar to those observed in previous studies $(7,30,33)$. However, the extent and severity of the lesions were dramatically affected by Se nutrition of the neonate. 
Table 5. Mean body and lung weights and Se concentrations, SeGPx activities, and histopathologic severity scores of lungs from cross-fostered (+) and negative control (-) pups reared in air by Se-deficient or Se-adequate dams

\begin{tabular}{|c|c|c|c|c|}
\hline & \multicolumn{2}{|c|}{ Se-deficient } & \multicolumn{2}{|c|}{ Se-adequate } \\
\hline & + & - & + & - \\
\hline Body wt $(\mathrm{g})^{*}$ & $8.78(0.54)$ & $9.96(0.54)$ & $9.06(0.25)$ & $8.63(0.36)$ \\
\hline \multicolumn{5}{|c|}{ (a) } \\
\hline $\mathrm{Wt}(\mathrm{g}) \dagger$ & $0.14(0.01)$ & $0.15(0.01)$ & $0.18(0.01)$ & $0.16(0.01)$ \\
\hline $\mathrm{Se}(\mathrm{nmol} / \mathrm{g})$ & $1.62(0.12)$ & $1.72(0.13)$ & $1.86(0.11)$ & $1.70(0.21)$ \\
\hline SeGPx $(U) \ddagger$ & $40.3(3.2)$ & $37.6(3.2)$ & $48.2(3.2)$ & $41.3(3.5)$ \\
\hline \multicolumn{5}{|l|}{$\begin{array}{l}\text { Histologic severity } \\
\text { scoresई }\end{array}$} \\
\hline $\begin{array}{l}\text { Interstitial in- } \\
\text { flammation }\end{array}$ & $0.12(0.08)$ & $0.15(0.11)$ & $0.12(0.08)$ & $0.07(0.07)$ \\
\hline $\begin{array}{l}\text { Septal attenua- } \\
\text { tion }{ }^{\dagger}\end{array}$ & $0.18(0.10)$ & $0.05(0.05)$ & $0.00(0.00)$ & $0.00(0.00)$ \\
\hline
\end{tabular}

$*$ Values are means for six litters $( \pm$ SEM).

$\dagger$ Significant effect of dietary $\mathrm{Se}(p<0.05)$ by analysis of variance.

$\$$ In $\mu$ mol NADPH disappearance $/ \mathrm{min} / \mathrm{g}$ protein.

$\S$ Each lesion received a score ranging from 0 (no lesion) to 3 (severe lesion) for the purpose of statistical analysis. Values are means for $15-$ 20 pups ( \pm SEM).

Yam et al. (30) reported that neonatal rats increase their pulmonary antioxidant enzyme activities (SOD, GPx, glutathione reductase) during oxygen exposure (1-5 d exposure), whereas adult rats do not show any significant increase. They suggested that the rapid increase in lung antioxidant enzymes in the neonatal rat might provide a mechanism for their increased tolerance to hyperoxia as compared with adults. However, Bucher and Roberts (33) did not find similar increases in antioxidant enzyme activities in neonatal rats during a similar period ( 1 to $3 \mathrm{~d}$ ) of oxygen exposure. In the latter experiment, it took 6 $\mathrm{d}$ of oxygen exposure to increase antioxidant enzyme activities. Sosenko et al. (34) noted in another experiment that GPx activity increased by $210 \%$, whereas SOD and catalase activities both increased by only $60 \%$ after $7 \mathrm{~d}$ of high oxygen exposure. In our experiment, the period of hyperoxia was $4 \mathrm{~d}$, and we saw an increase in SeGPx activity only. Results of this and previous studies imply that $4 \mathrm{~d}$ of hyperoxia in a neonatal rat is insufficient to induce an increase in the activities of antioxidant enzymes such as SOD, non-SeGPx, and catalase, but sufficient to induce SeGPx, and that SeGPx may be a more sensitive indicator of oxidant stress than either SOD, non-SeGPx, or catalase.

The only well-described biochemical role of Se is as an integral component of the enzyme SeGPx. Cross et al. (35) showed that $35 \%$ of adult rats fed Se-deficient diets $(0.02 \mathrm{ppm})$ died $3 \mathrm{~d}$ after oxygen (80\%) exposure. All rats fed Se-supplemented diets $(0.5$ $\mathrm{ppm}$ ), however, survived during that period. Forman et al. (36) also demonstrated a significant difference in the $\mathrm{LD}_{50}$ response between Se-deficient and Se-adequate adult rats under conditions of hyperoxia. Both investigators ascribed the higher oxygen resistance of Se-adequate adult rats to higher SeGPx activity. In our study, using neonatal rats, no differences in the antioxidant enzyme activities including SeGPx were found between Sedeficient and Se-adequate pups under oxygen exposure. However, only Se-adequate pups were protected from oxygen-induced lung damage. These results suggest that Se may have a unique, as yet unspecified role in the developing neonatal lung, in addition to its known role as an essential component of SeGPx.

It is possible that human LBW infants with only minimal body stores of Se could experience increased lung damage during oxygen treatment similar to that of the Se-deficient pups. Infant BPD, which is directly related to high oxygen ventilation, progresses from pulmonary edema and inflammation in early stages to chronic fibrosis, and emphysema. The interstitial inflammation found in this study, therefore, resembles the acute histologic lesions found in infants with early BPD. Marginal Se deficiency in LBW infants, therefore, may have a large impact on lung development and response of the lung to oxidant stress when supplemental oxygen support is needed.

Some possible roles for $\mathrm{Se}$ in protection against oxygeninduced lung damage may be considered. It is possible that Se turnover in the lung is more rapid under conditions of oxidant stress. Se-adequate pups, having a greater store of liver Se (Table 4), could supply the necessary Se rapidly from the liver. Therefore, they would be able to dispose of excess reactive oxygen metabolites in the lung before severe damage occurred by some as yet undefined mechanism. In contrast, Se-deficient rats would not have enough reserve $S e$ available to rapidly mount a response to detoxify reactive oxygen intermediates in the lung. The higher Se concentrations in the liver of the Se-adequate pups in our study, and the even higher stores of liver Se in the Se-adequate air group, lend support to this possibility.

In summary, results from our study showed a decrease in the lung weight and presence of pulmonary septal attenuation of pups under conditions of Se inadequacy. Although there was an increase in specific SeGPx in both Se-deficient and Se-adequate pups under oxygen exposure, only Se-adequate pups were protected from severe lung lesions. Therefore, these data indicate that Se has an important role in the development of neonatal lungs, which is magnified under conditions of hyperoxia. The protective effect of Se under conditions of hyperoxia cannot be completely attributed to its role as a component of the antioxidant enzyme SeGPx, but to some other as yet undetermined function of Se. Further investigations are necessary to establish the role of Se in the development of neonatal lung and possible clinical significance in LBW infants.

Acknowledgments. The authors thank Dr. Wanda HaschekHock for scientific advice and encouragement, Laura Brady and Janice Rutherford for technical assistance, Tonja Henze for animal care, and the Word Processing Center, College of Veterinary Medicine, and Nancy Hopkins, Department of Nutrition, for secretarial assistance.

\section{REFERENCES}

1. Combs GF, Combs SB 1986 The Role of Selenium in Nutrition. Academic Press, Orlando, FL, pp 265-326

2. Lockitch G, Jacobson B, Quigley G, Dison P, Pendray M 1989 Selenium deficiency in low birth weight neonates: an unrecognized problem. J Pediatr 114:865-870

3. Amin S, Chen SY, Collipp PJ, Castro-Magana M, Maddaiah VT, Klein SW 1980 Selenium in premature infants. Nutr Metab 24:331-340

4. Stark AR, Frantz III ID 1986 Respiratory distress syndrome. Pediatr Clin N Am 33:533-544

5. Northway Jr WH, Rosan RC, Porter DY 1967 Pulmonary disease following respiratory therapy of hyaline-membrane disease: bronchopulmonary dysplasia. N Engl J Med 276:357-368

6. Bancalari E, Gerhardt T 1986 Bronchopulmonary dysplasia. Pediatr Clin North Am 33:1-23

7. Frank L, Bucher JR, Roberts RJ 1978 Oxygen toxicity in neonatal and adult animals of various species. J Appl Physiol 45:699-704

8. Crapo JD, Tierney DF 1974 Superoxide dismutase and pulmonary oxygen toxicity. Am J Physiol 226:1401-1407

9. Kimball RE, Reddy K, Peirce TH, Schwartz LW, Mustafa MG, Cross CE 1976 Oxygen toxicity: augmentation of antioxidant defense mechanisms in rat lung. Am J Physiol 230:1425-1431

10. Freeman BA, Topolosky MK, Crapo JD 1982 Hyperoxia increases oxygen radical production in rat lung homogenates. Arch Biochem Biophys 216:477-484

11. Lawrence RA, Burk RF 1976 Glutathione peroxidase activity in seleniumdeficient rat liver. Biochem Biophys Res Commun 71:952-958

12. Jenkinson SG, Lawrence RA, Burk RF, Gregory PE 1983 Non-selenium dependent glutathione peroxidase activity in rat lung: association with lung glutathione-s-transferase activity and the effects of hyperoxia. Toxicol Appl Pharmacol 68:399-404

13. Rotruck JT, Pope AL, Ganther HE, Swanson AB, Hafeman DG, Hoekstra WG 1973 Selenium: biochemical role as a component of glutathione peroxidase. Science 179:588-590

14. World Health Organization 1987 Environmental Health Criteria 58: Selenium World Health Organization, Geneva

15. Smith AM, Picciano MF 1986 Evidence for increased selenium requirement for the rat during pregnancy and lactation. J Nutr 116:1068-1079 
16. Underwood EJ 1977 Trace Elements in Human and Animal Nutrition, 4th Ed. Academic Press, New York

17. Frank L, Sosenko IRS 1987 Prenatal development of lung antioxidant enzymes in four species. J Pediatr 110:106-110

18. Burk RF 1983 Biological activity of selenium. Annu Rev Nutr 3:53-70

19. Frank L 1985 Effect of oxygen on the newborn. Fed Proc 44:2328-2334

20. Roberts RJ 1979 Employment of pulmonary superoxide dismutase, catalase, and glutathione peroxidase activity as criteria for assessing suitable animal models for studies of bronchopulmonary dysplasia. J Pediatr 95:904-909

21. National Research Council 1978 Nutrient Requirements of Laboratory Animals, No 10, 3rd Rev Ed. National Academy of Sciences, Washington, DC pp 7-37

22. McCarthy TP, Brodie B, Milner JA, Bevill RF 1981 Improved method for selenium determination in biological samples by gas chromatography. J Chromatogr 225:9-16

23. Paglia DE, Valentine WN 1967 Studies on the quantitative and qualitative characterization of erythrocyte glutathione peroxidase. J Lab Clin Med 70:158-169

24. Levander OA, DeLoach DP, Morris VC, Moser PB 1983 Platelet glutathione peroxidase activity as an index of selenium status in rats. $J$ Nutr $113: 55-63$

25. McCord JM, Fridovich I 1969 Superoxide dismutase: an enzymic function for erythrocuprein (hemocuprein). J Biol Chem 244:6049-6055

26. Holmes RS, Masters CJ 1970 Epigenetic interconversions of the multiple forms of mouse liver catalase. FEBS Lett 11:45-48
27. Lowry OH, Rosebrough NJ, Farr AL, Randall RJ 1951 Protein measurement with the Folin phenol reagent. J Biol Chem 193:265-275

28. Steel RGD, Torrie JH 1980 Principles and Procedures of Statistics: A Biometrical Approach, 2nd Ed. McGraw-Hill, New York

29. SAS Institute Inc 1985 SAS User's Guide: Statistics, 5th Ed. SAS (Statistical Analysis System) Institute, Cary, NC, pp 434-506

30. Yam J, Frank L, Roberts R 1978 Oxygen toxicity: comparison of lung biochemical responses in neonatal and adult rats. Pediatr Res 12:115-119

31. Crapo JD, Barry BE, Foscul HA, Shelburne J 1980 Structural and biochemical changes in rat lungs occurring during exposures to lethal and adaptive doses of oxygen. Am Rev Respir Dis 122:123-143

32. Rosenbaum RM, Wittner M, Lenger M 1969 Mitochondrial and other ultrastructural changes in great alveolar cells of oxygen-adapted and poisoned rats. Lab Invest 20:516-528

33. Bucher JR, Roberts RJ 1981 The development of the newborn rat lung in hyperoxia: a dose response study of lung growth, maturation, and changes in antioxidant enzyme activities. Pediatr Res 15:999-1008

34. Sosenko IRS, Innis SM, Frank L 1988 Polyunsaturated fatty acids and protection of newborn rats from oxygen toxicity. J. Pediatr 112:630-637

35. Cross CE, Hasegawa G, Reddy KA, Omaye ST 1977 Enhanced lung toxicity of oxygen in selenium-deficient rats. Res Commun Chem Pathol Pharmaco 16:695-706

36. Forman HJ, Rotman EI, Fisher AB 1983 Roles of selenium and sulfurcontaining amino acids in protection against oxygen toxicity. Lab Invest 49:148-153

\section{Announcement}

\section{Seeking Acute Cases of Rheumatic Fever and Sydenham's Chorea}

The Child Psychiatry Branch of the National Institute of Mental Health is seeking patients for a study of the course of psychologic symptoms accompanying Sydenham's chorea and rheumatic fever. Eligible patients should have had recent (within 2 months) onset of rheumatic fever, continue to have symptoms, and be at least 6 years of age. This study will rate a variety of psychologic and psychiatric symptoms and link these symptoms to antiCNS autoantibodies. The results will be important in defining the etiology of childhood-onset psychiatric disorders. Patients and their parents will be asked to travel at NIH expense to the NIMH in Bethesda, MD for an initial interview. Brief follow-up interviews will be conducted by telephone every 2 months for 1 year. Serum samples $(5 \mathrm{~mL})$ will be obtained on four separate occasions. There will be no expense to the patient and no remuneration. Please call Dr. Susan Swedo at (301) 496-6081, or write: Dr. Swedo, Child Psychiatry Branch, NIMH, Bldg. 10, Room 6N240, 9000 Rockville Pike, Bethesda, MD 20891. 